\title{
Redes sociales en la enseñanza superior
}

\author{
Alícia Martí Climent, Pilar Garcia Vidal
}

Universitat de València, alicia.marti@uv.es, pilar.garcia-vidal@uv.es

\begin{abstract}
In this communication we expect to show project work experiences using social networks (Facebook, Twitter, Instagram and Youtube) as an educational resource in higher education to share, create, inform and communicate. In this way, educational innovation has been promoted with new work methods improving university students' motivation and academic results in classroom work.
\end{abstract}

Keywords: TIC, Social Networks, Higher Education, Educational Innovation.

\begin{abstract}
Resumen
En esta comunicación pretendemos mostrar experiencias realizadas con proyectos de trabajo que emplean las redes sociales (Facebook, Twitter, Instagram y Youtube) como recurso educativo en la enseñanza superior para compartir, crear, informar y comunicar. De esta manera, se favorece la innovación educativa con nuevos medios que suponen diferentes metodologías y mejoran la motivación del alumnado universitario y el resultado del trabajo en el aula.
\end{abstract}

Palabras clave: TIC, redes sociales, educación superior, innovación educativa.

\section{Introducción}

Esta comunicación tiene como objetivo presentar el uso de las redes sociales como recurso educativo en la enseñanza superior con el fin de animar al profesorado a la utilización de este tipo de medios en su práctica didáctica.

Actualmente la universidad acoge alumnado de la llamada generación en red (Generation red, Tapscott, 2009) o millenials ${ }^{1}$, que está acostumbrado a la interactividad que promueven

\footnotetext{
${ }^{1}$ Como apuntan Meso Ayerdi, Pérez Dasilva y Mendiguren Galdospin (2011), las nuevas generaciones han recibido varias etiquetas, como Generation Red (Tapscott, 1998), e-
} 
las redes sociales. La influencia del audiovisual y de Internet está muy presente en estos nativos digitales (Prensky, 2001). Al estar más familiarizados con el uso de las TIC tienen un concepto positivo sobre su utilidad en el aprendizaje.

Las herramientas tecnológicas que les permiten compartir, crear, informar y comunicarse son múltiples. Las redes sociales se han convertido en esenciales en sus vidas. Actualmente, los jóvenes las utilizan con frecuencia (sobre todo Twitter e Instagram) con fines diversos. Así pues, a partir de este hecho nace nuestra propuesta didáctica: el uso de las redes sociales como recurso educativo en la enseñanza superior.

Nuestra experiencia se basa en la aplicación de este recurso de las nuevas tecnologías de la información y la comunicación (TIC) desde la perspectiva pedagógica de las técnicas del aprendizaje colaborativo y la creación de conocimiento en red, es decir, de las tecnologías para el aprendizaje y el conocimiento (TAC). El uso de estas herramientas tecnológicas debe seguir siempre principios pedagógicos didácticos con el fin de conseguir la innovación educativa adecuada en los procesos formativos. La innovación y la mejora de las prácticas educativas mediante las tecnologías depende del buen uso en su aplicación didáctica. La renovación metodológica debe tener en cuenta los avances en didáctica de la lengua y la literatura y debe incorporar las posibilidades de la red para investigar, reflexionar y compartir (Manresa, Durán y Ramada, 2012).

Concretamente, hemos utilizado las redes sociales en la asignatura Complementos para la formación disciplinar de la especialidad de lengua y literatura catalana dentro del Máster universitario en profesorado de educación secundaria de la Universitat de València. A partir de los datos recogidos en unos cuestionarios realizados por el alumnado del Máster desde el curso 2011-12 hasta el 2017-18, constatamos el tipo de recursos TIC que emplean los alumnos para la elaboración y la presentación de trabajos académicos de diferentes géneros. Observamos un aumento evidente en los últimos años de la diversidad de herramientas utilizadas por el alumnado para trabajar diferentes géneros académicos. Las plataformas digitales más utilizadas para publicar y difundir los trabajos multimedia son: blogs (Blogger, Wordpress), wiki (Wikispaces), webquest (Google Sites), redes sociales (Twitter, Facebook, Instagram, Tuenti), servidores para alojar presentaciones (Slideshare, Scribd), edición de revistas digitales (Issuu), participación en una enciclopedia digital (Wikipedia), elaboración de rutas en un mapa digital (Goggle Maps), compartir vídeos en red (Youtube) y presentaciones en línea (Prezi).

En este trabajo nos centraremos en el uso de las redes sociales en proyectos realizados por los alumnos del Màster de Secundaria con una finalidad didáctica, como ejemplos de experiencias que pueden servir como modelos de trabajos académicos con un buen uso de este tipo de recursos.

Generation, Millennial Generation, Generación Interactiva (Bringué y Sadaba, 2008) o Generación Einstein (Piscitelli, 2009).

(cc) EY-NC-ND 2018, Universitat Politècnica de València

Congreso IN-RED (2018) 


\subsection{Las redes sociales}

Las redes sociales son sitios web que permiten compartir de manera virtual lo que hacemos cotidianamente (actividades diversas) y también lo que creamos encontramos en la red (vídeos, música, imágenes, etc). Como señala Macías (2016: 228), "Las redes sociales, entendidas como comunidad abierta y no jerarquizada, se basan en el principio de las tres ces: contenido, construcción y colaboración (content, construction, colaboration)".

De entre las diversas redes sociales en línea, Área (2008) distingue tres tipos: redes de masas (Facebook, Twitter,...) en que las personas usuarias comparten un perfil, fotos, comentarios, etc.; redes abiertas para compartir archivos en diferentes formatos (YouTube, Slideshare, Flickr...) y redes temáticas o comunidades de aprendizaje con un objetivo común o un interés específico (Ning, Elgg...). En el uso de las redes sociales en el Máster de Secundaria han ido apareciendo cambios desde el curso 2010-11 al 2017-18 por lo que se refiere al tipo de plataformas utilizadas. Han ido subtituyéndose las que se utilizaban como redes temáticas para el seguimiento del trabajo de clase, desde Ning, Wikispaces a Google Sites; han augmentado el uso de las redes de masas, como Facebook, Twitter, Instagram; y en las redes abiertas, ha predominado YouTube.

\subsection{El uso académico de las redes sociales}

Cualquier red social permite mantener el contacto entre las personas. El uso de las redes sociales favorece el desarrollo de las habilidades lingüísticas y comunicativas mediante el hipertexto que se configura con la combinación de códigos verbales gráficos y sonoros, y la interacción comunicativa que se genera a través de la conversación y el debate en la red. Cada usuario se convierte en emisor y receptor de textos diversos. La utilización de las tecnologías de la información y la comunicación resulta un elemento esencial para informarse, aprender y comunicarse. (Garcia y Martín, 2010)

Posiblemente una de las redes usadas con fines educativos más habitual sea Ning, como podemos ver en las experiencias de Haro (2009) y García y Martí (2010), aunque ha disminuido su uso desde que dejó de ser gratuita. Últimamente encontramos destacadas aplicaciones didácticas para el trabajo de la lectura social y colaborativa mediante Twitter (Lluch, 2014; Rovira, 2015). La lectura se comparte, se comenta y se muestra. Según Lluch (2014), la clave del éxito radica en "su carácter social, público, interactivo y global que permite socializar la lectura, compartirla con cualquier otro adolescente de cualquier lugar del mundo y a cualquier hora" (Lluch, 2014: 19).

Así pues, las redes sociales o, según los expertos, servicios 2.0 con características de red social, en el ámbito educativo presentan diferentes posibilidades didácticas. De acuerdo con Haro $(2009,2010)$ y Orihuela (2009), las redes sociales en línea permiten generar nuevas sinergias entre los miembros de una comunidad educativa, ya que favorecen la comunicación bidireccional entre el alumnado y el profesorado, y por tanto el efecto de proximidad y el aumento de la fluidez y sencillez de la comunicación. Pero, además, proyectan y consolidan las relaciones interpersonales una vez que han terminado los estudios. También facilitan la circulación de información, la organización de eventos, el 
compartir recursos y la coordinación y trabajo de varios grupos de aprendizaje (clase, asignatura, grupo de alumnos de una asignatura, etc.) mediante la creación de los grupos apropiados.

Sobre el uso las redes sociales en la educación, Pérez García (2013) señala que es recomendable su uso ya que favorecen la adquisición de las competencias tecnológicas, fundamentales tanto en el ámbito educativo como en el laboral. Garcia y Martí (2016) apuntan que éstas facilitan una planificación didáctica eficaz y motivadora para el alumnado, potencian la creatividad y la originalidad, fomentan el trabajo colaborativo, refuerzan el aprendizaje individual y permiten la difusión de propuestas de trabajo innovadoras.

El uso de las redes sociales presenta una ventaja fundamental: el acercamiento del aprendizaje formal y el informal. Sin embargo, este aspecto conlleva al mismo tiempo la necesidad de enseñar a los alumnos a participar, ya que pueden tender a utilizarlos sólo de forma personal. Es necesario, por lo tanto, enseñar a a usar la red como herramienta para el trabajo académico y para potenciar la colaboración (Garcia y Martí, 2010).

Gómez, Roses y Farias (2012) señalan que entre las actividades que realiza el alumnado en las redes sociales encontramos: resolver dudas sobre la materia, mantenerse informados del ritmo de las clases y realizar trabajos en grupo; si bien, ninguna de estas tareas es demasiado frecuente. Los autores apuntan también que las redes sociales resultan más rentables en contextos en que se utilizan las metodologías activas y participativas, que consiguen, entre otros, fomentar el trabajo colaborativo, el aprendizaje continuo, la cohesión del grupo, el aprendizaje autónomo, el diálogo y la comunicación entre los alumnos y entre éstos y el profesor, el pensamiento crítico.

Por otra parte, el estudio de Gómez, Roses y Farias (2012) sobre el uso académico que hacen los universitarios de las redes sociales, a partir de una encuesta a una muestra representativa de estudiantes de la Universidad de Málaga, plantea una paradoja: aunque los alumnos utilizan frecuentemente las redes sociales, su uso para actividades académicas es escaso y entre sus contactos no suelen tener a ningún profesor.

En nuestro trabajo, nos llama la atención la escasa formación en TIC que demuestra la mayoría de los estudiantes del Máster, salvo en el caso de los que han cursado una especialidad que incluye trabajar con varias tecnologías, como en el caso de los grados en comunicación audioviosual o periodismo. De ello se deduce que la incorporación de las TIC no tiene la relevancia que debería tener en la formación universitaria de algunas especialidades, por lo que conviene considerarla para completar la formación de los futuros docentes, que deben saber utilizar las TIC como herramientas de trabajo habituales e incorporarlas en el aprendizaje continuo a lo largo de la vida, tanto personal como profesional (Martí y Garcia, 2014). Hay que añadir que uno de los obstáculos para el uso de las redes sociales en la enseñanza son también las restricciones en el acceso a las redes sociales en algunos centros educativos.

(c)) EY-NC-ND 2018, Universitat Politècnica de València

Congreso IN-RED (2018) 


\section{Objetivos}

Nos proponemos potenciar el uso de las redes sociales en trabajos académicos de enseñanza superior con el objetivo de suplir estos obstáculos que hemos observado durante nuestra experiencia académica como profesoras del ámbito universitario y de enseñanza secundaria. El poco uso que aún se hace de estas herramientas con fines didácticos supone que no se aproveche suficientemente su potencial a nivel de motivación e implicación del alumnado, de posibilidades para aumentar el grado de interacción y de colaboración entre profesorado y alumnado, de difusión y publicación inmediata del trabajo realizado en el aula.

Además, planteamos como objetivos específicos facilitar la innovación en proyectos con redes sociales que permitan nuevos planteamientos para trabajar con una metodología más colaborativa y participativa, que aprovechen de manera más efectiva los recursos más actualizados de la red.

Por otra parte, aprender a hacer un uso adecuado de las redes sociales permite tener en cuenta estos recursos que pueden resultar muy productivos desde el punto de vista educativo para el aprendizaje y la comunicación. Las redes sociales permiten desarrollar la capacidad lectora, la expresión oral y la creación de textos escritos multimedia del alumnado.

Finalmente, con nuestro trabajo pretendemos mostrar experiencias realizadas con proyectos de trabajo que emplean las redes sociales con buenos resultados a nivel académico.

\section{Desarrollo de la innovación}

Presentamos ejemplos de proyectos realizados con algunas de las redes más utilizadas en educación, como Facebook, Twitter, Instagram y YouTube, que forman parte de experiencias que hemos llevado a cabo en el aula con el alumnado del Máster de Secundaria.

\subsection{Facebook}

Esta red es un punto de encuentro colectivo y atemporal. El muro de Facebook permite a cada usuario disponer de un espacio para difundir contenidos textuales, visuales, audiovisuales y de audio entre los miembros de la red con los que el usuario ha aceptado compartir estos contenidos. Ahora bien, Facebook no está concebido como una plataforma docente. Encontramos diversas dificultades para su uso en la docencia, como no poder hacer un seguimiento personalizado de la actividad de cada usuario y la imposibilidad de compartir documentos en PDF.

Sin embargo, un estudio de Gómez, Roses y Farias (2012) apunta que la red social más adecuada para el uso académico es Facebook, por la cantidad de funciones que presenta (textos, vídeos, foros, chats, etc.) y por la creación de contenidos. De hecho, los autores 
recomiendan la creación de un grupo en Facebook con el nombre de la asignatura por parte del profesor/a.

Como ejemplo de experiencia educativa de uso de esta red, tenemos la creación en el Máster de Secundaria del programa de radio El redolí que dispone de perfiles del programa en las redes sociales Facebook y Twitter. El objetivo de emplear estas redes es ayudar a difundir el programa y potenciar la interacción con el oyente. Además, existe la voluntad de construcción de una comunidad de aprendizaje alrededor del programa, un objetivo para el que es necesario potenciar la participación. En este sentido, y sólo a título de ejemplo, podemos destacar la propuesta de llevar a cabo la participación en un concurso que se realiza en el programa mediante estas dos redes sociales.

Otro proyecto que emplea las redes sociales Facebook y Twitter es la creación del periódico L'Interrogant que, además de una edición de papel, tiene una edición digital en una web y interacciona con sus lectores mediante estas redes sociales.

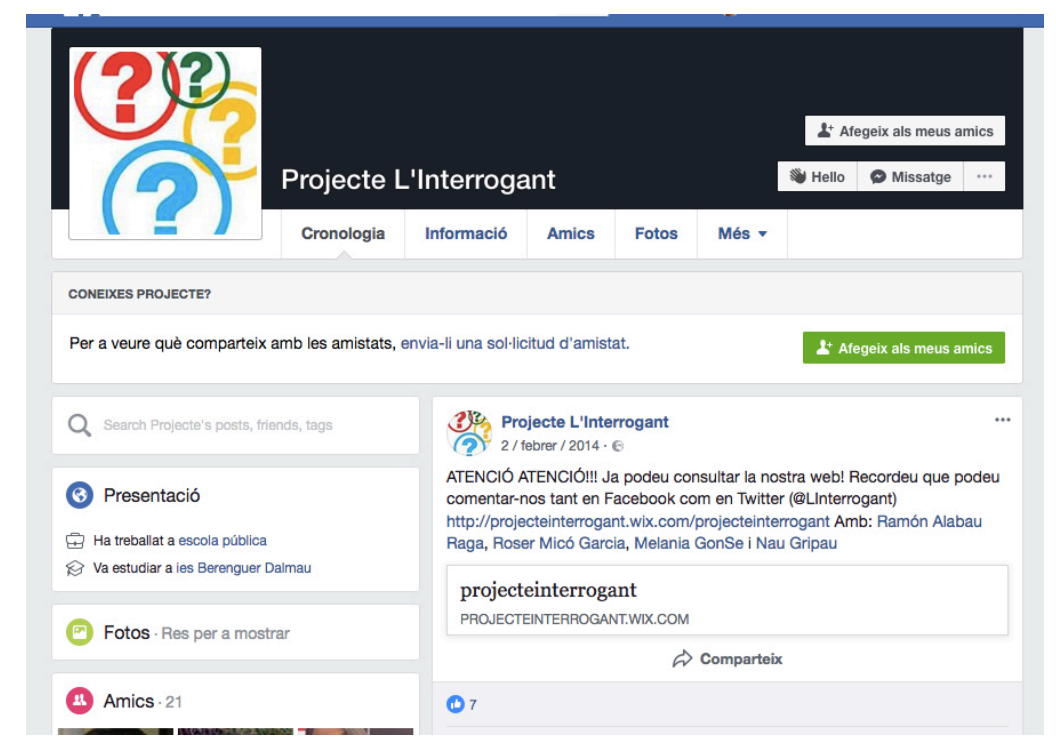

Fig. 1 Pàgina de Facebook del periódico L'Interrogant

\subsection{Twitter}

Con respecto a esta red, encontramos la ventaja de la limitación en el uso de caracteres que desarrolla la capacidad de síntesis. Sobre Twitter, Lluch (2012) apunta su uso como espacio de comunicación en torno a libros, autores o lecturas. Según la autora, podemos aprovechar las características de Twitter para:

- informar sobre el libro que leemos: los avances que hacemos, las partes que nos gustan, escribir una frase que en guste, etc. 
- iniciar una conversación sobre un libro, una temática o un autor

- comentar noticias relacionadas con la lectura o los libros que leeremos

- compartir enlaces de Internet que nos informan sobre los temas lectores que nos interesan

- pedir ayuda sobre un libro que no encontramos, un capítulo que no entendemos, etc.

(Lluch, 2012: 203)

En relación al uso académico de Twitter, Lluch y Nicolás (2015) hacen algunas recomendaciones a partir de los trabajos de Torres (2011), Zayas (2012), Sáez (2013), Millán (2013) y Lluch (2014), como la importancia de aportar valor a la comunicación, de interactuar con los usuarios que comparten intereses y etiquetas y de hacer un uso adecuado, correcto y normativo de la lengua, entre otros. Usar Twitter "de manera adecuada, con registro formal y propósito académico" para "aprender y ampliar los horizontes del espacio académico en cualquier lugar o momento" (Lluch, Nicolás, 2015: 201-261).

Sobre el uso que se hace de la red Twitter a nivel educativo, encontramos ejemplos para trabajar lecturas, como la experiencia desarrollada por la Universitat de València Diàleg entre l'escriptor $i$ els lectors joves, organizado desde 2002 por la profesora Carme Gregori. El profesor Ignasi Moral participa con las tertulias literarias de sus alumnos mediante Twitter mientras se lleva a cabo la lectura (Lluch, 2012: 155-159), utilizando una etiqueta para compartir sus comentarios sobre el libro y consensuando una serie de normas sobre el tipo de comentarios, la forma, los plazos de lectura, etc.

Este tipo de tertulia literaria con Twitter está implementado también en el Máster de Secundaria para hacer comentarios, citas, reflexiones, enlaces a documentos, webs, etc. sobre el libro que se lee. Se parte de una cuestión antes de la lectura para incluir impresiones previas que se insertan en una etiqueta con el fin de interactuar entre los alumnos y el profesor durante la lectura. Posteriormente, se destacan los tuits de interés y se realiza un debate sobre las ideas comentadas por los lectores. 


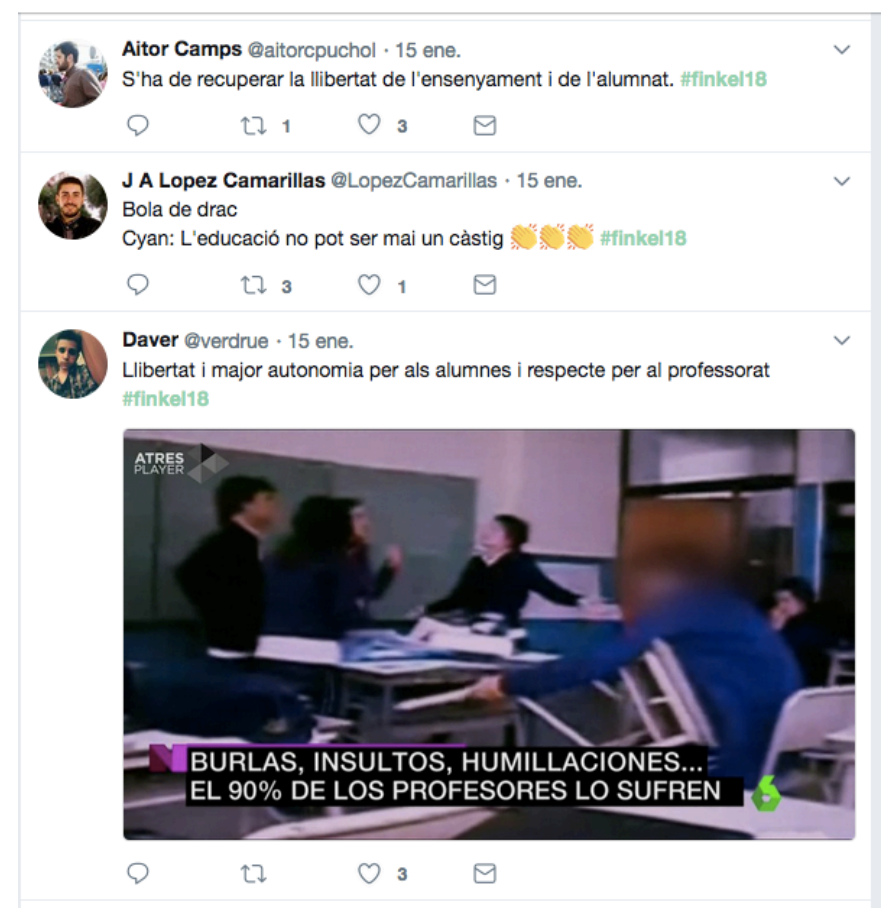

Fig.2 Tuits con la etiqueta del libro comentado por el alumnado del Máster

Otra experiencia para trabajar la lectura con Twitter, propuesta en el Máster de Secundaria, consiste en seguir la lectura de la obra Aloma de Mercè Rodoreda mediante tuits con aquellas palabras y frases de la obra que les resultan más impactantes al alumnado y les provocan algún sentimiento, emoción o pensamiento, tanto en relación a la variedad dialectal como con la manera de expresarse de los personajes, lo que sienten y lo que hacen sentir y reflexionar a los lectores. Se utilizarían tres tipos de etiquetas: una para el léxico, como \#LèxicAloma; otra para los pensamientos y las visiones de los personajes, como \#PensemAloma; y otra para los pensamientos, emociones y opiniones que les sorprenden a los alumnos mientras leen, o para hacer de amigos de Aloma, a quien aconsejan, como \#JoSócAloma. La elección de etiquetas se dejará en manos del alumnado. El profesorado tiene acceso directo a la cronología de publicaciones de la actividad.

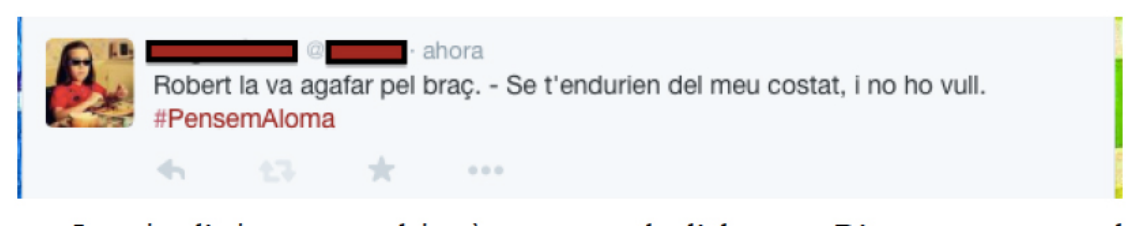

Fig. 3 Tuit con la etiqueta del comentario de los alumnos sobre la novela Aloma 


\subsection{Instagram}

Esta red permite hacer fotografías, modificarlas con efectos especiales y compartirlas, y, además, desde hace poco tiempo, podemos hacer vídeos de un máximo de 15 segundos de duración, a los que se les puede aplicar diferentes filtros. Actualmente es una de las redes sociales con más éxito, por lo que se podría aprovechar desde la enseñanza.

Un ejemplo de su uso en el ámbito educativo es el World Mobile City Project (WMCP), un proyecto colaborativo innovador, impulsado por la Red Lacenet, con el objetivo de que el alumnado conozca la ciudad con herramientas de geolocalización (Google Maps), códigos QR y realidad aumentada, mediante el uso del móvil, en el que se utiliza Instagram. La Asociación de profesorado lentretants organiza desde hace años la Valencianada (\#VLCda) en la ciudad de Valencia, una actividad multidisciplinar dirigida preferentemente a alumnos de $2^{\circ}$ ciclo de secundaria y de bachillerato que, en grupos reducidos, deben localizar, mediante sus dispositivos móviles y el uso de determinadas aplicaciones, varios puntos de interés social, literario, científico, artístico, histórico... de la ciudad de València. Simultáneamente, deben responder cuestiones relacionadas con los lugares que visitan y hacer difusión a través de diversas redes sociales, concretamente deben aportar en Instagram cuatro imágenes y un breve vídeo, cubriendo todos los puntos, todas ellas con explicaciones de calidad.

Otro ejemplo de utilización didáctica lo tendríamos en algunos proyectos desarrollados en el Máster de Secundaria, como el llamado \#Instacacera, que tiene como objetivo principal introducir al alumnado del segundo ciclo de Educación Secundaria Obligatoria en el aprendizaje y la identificación de neologismos, préstamos léxicos y barbarismos. La secuencia didáctica propuesta consiste en la elaboración de un álbum digital en la red social Instagram. Cada alumno deberá realizar una búsqueda de neologismos, barbarismos y préstamos léxicos. Con estas palabras tendrá que hacer una fotografía con la palabra en concreto que en el momento puntual está trabajando para analizarla y encontrar su equivalente al catalán siguiendo la siguiente estructura:

- Pie de foto: explicación del error que ha encontrado y su corrección.

- Inclusión del enlace donde ha encontrado la corrección (diccionario en línea, libro, gramática, vocabulario, etc.).

- Especificación del lugar donde ha encontrado la palabra analizada adjuntando la localización. 


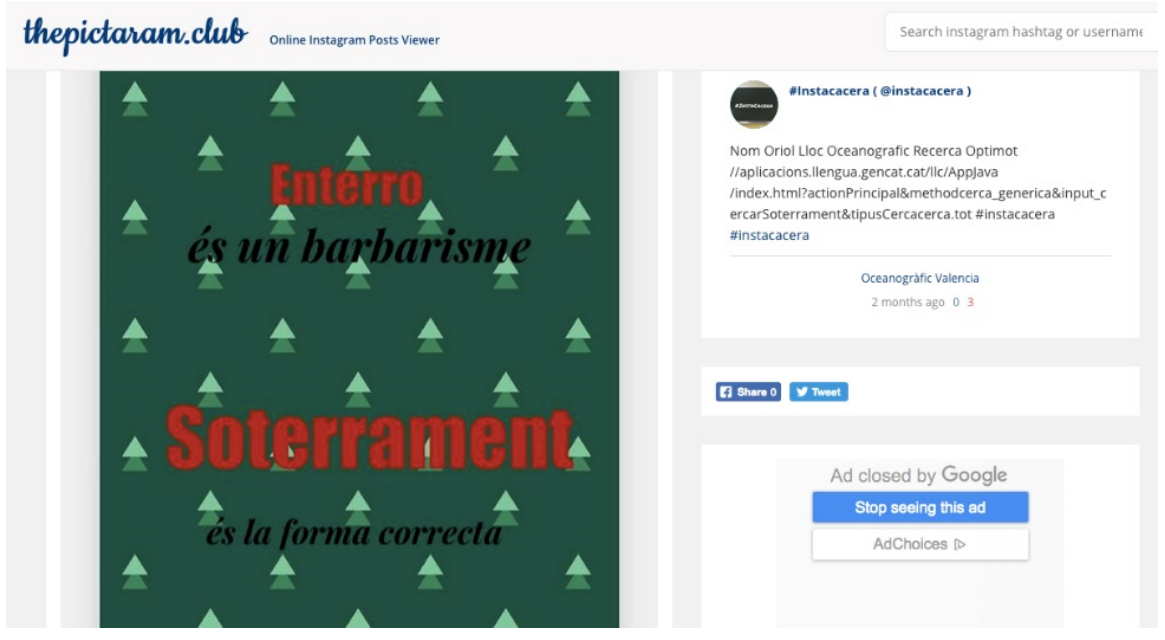

Fig. 4 Imagen de Instagram del proyecto \#Instacacera

\subsection{YouTube}

Como señala Martí (2018), Internet se ha convertido en uno de los principales medios de difusión de contenidos y concretamente Youtube es uno de los portales que ha aumentado más su uso en los últimos años gracias a su utilización por muchos jóvenes como medio de comunicación, plataforma de expresión sobre temas diversos, lo que ha llevado al nacimiento del fenómeno conocido como YouTubers, algunos de los cuales podríamos decir que son estrellas mediáticas, incluso de alcance mundial. A la sombra de esta figura ha crecido la comunidad de los booktubers o bibliotubers, jóvenes que quieren compartir su pasión por los libros haciendo vídeos divertidos.

También destaca la proliferación del booktrailer para potenciar la lectura en educación, como en el caso del concurso de booktrailer Martí Sorolla, con el objetivo de promover la lectura utilizando la creatividad y el uso de las TIC para elaborar un vídeo-resumen del libro que han leído, acercando la lectura con un formato más próximo a nuestros adolescentes; o la convocatoria que se hace en los premios Sambori, entre otros.

Desde hace tiempo el vídeo está presente en las aulas pero, como apunta García García (2014), su utilización como recurso educativo ha vivido una evolución vertiginosa en los últimos años. Según el autor, el uso educativo del vídeo debería enfocarse más hacia la producción que no al simple visionado o consumo: "deberíamos aprovechar todo el potencial multimedia de los dispositivos móviles y el potencial creativo de nuestro alumnado, y potenciar su papel como prosumidor y generador de contenidos audiovisuales" (García García, 2014: 117).

Como ejemplo de uso de esta red, tomamos el proyecto Dialectes en xarx@, desarrollado en el Máster de Secundaria. Lo que se pretende con esta secuencia didáctica es introducir las principales variedades diatópicas del catalán de la forma más amena posible, con la 
ayuda de las nuevas tecnologías. En este proyecto los alumnos deben registrar en el canal de Youtube, creado para la clase, la lectura de los fragmentos en la variedad dialectal que han trabajado.

Otro ejemplo de proyecto desarrollado por el alumnado del Máster de Secundaria donde se emplea la difusión de una producción audiovisual en Youtube, es la creación de un "videoling" (vídeo lingüístico), que consiste en crear un noticiario didáctico para trabajar cuestiones lingüísticas. El noticiario consta de tres partes, en que los alumnos tienen que grabar una serie de vídeos simulando las diferentes secciones de los informativos. La primera se explicará a partir de la sección del tiempo y tratará de cuestiones de sintaxis con el análisis de una serie de titulares. En segundo lugar, se dedica unos minutos a un "anuncio-denuncia", en el que se destaca algún tema de problemática social y se trabaja el eslogan publicitario. Por último, se corrigen errores ortográficos de algunos textos que aparecen en fotografías de lugares de la ciudad donde los alumnos han encontrado estos errores.

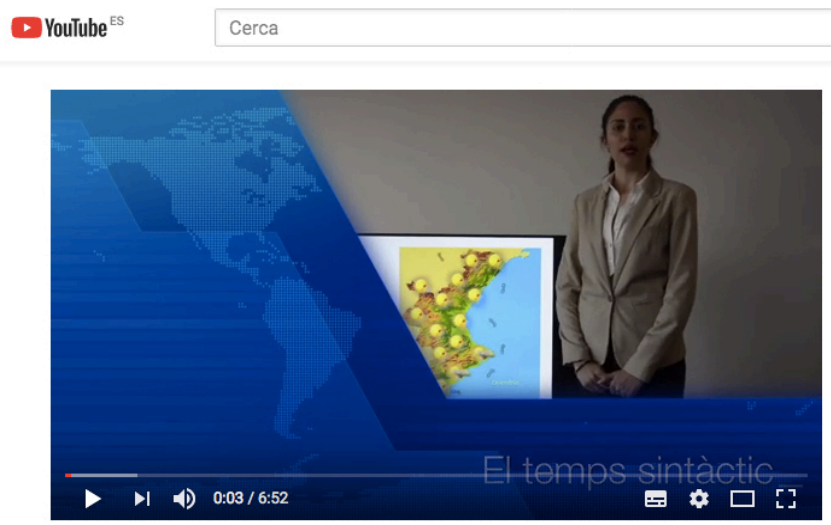

VIDEOLING

53 visualitzacions

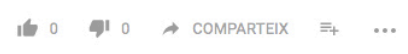

Fig. 5 Imagen del noticiario didáctico realizado por los alumnos del Máster

\section{Resultados}

Hemos comprobado, a partir de los cuestionarios contestados por los alumnos y de los proyectos realizados desde el curso 2011-12 hasta el 2017-18, un aumento del interés de los estudiantes por usar las redes sociales en proyectos para trabajar la lengua y la literatura en Secundaria. Con estas herramientas se ha favorecido la innovación educativa proponiendo nuevas formas de trabajar que mejoran la motivación y el resultado del trabajo en el aula. 
El alumnado cada vez tiene un mayor grado de conocimiento de recursos TIC y la posibilidad de incluir las redes sociales en la docencia favorece el trabajo colaborativo y la posibilidad de compartir documentos en diferentes formatos. Por otra parte, destacamos las aportaciones de las redes sociales a la escritura académica ya que suponen la aparición de nuevos géneros que implican:

- nuevas formas de escritura más sintética e inmediatez en la difusión de los textos: el post en Facebook, el tuit en Twitter, el pie de foto en Instagram,

- un carácter más público, lo que implica la posibilidad de compartir, revisar y cuidar más la presentación de los textos,

- una mayor exigencia en la elaboración de los proyectos ya que tienen más difusión,

- una necesaria coordinación que potencia el trabajo colaborativo, la interacción y la reflexión entre los estudiantes.

\section{Conclusiones}

Con el uso de las redes sociales se favorece el desarrollo de diferentes competencias (comunicativa, lingüística, digital, tratamiento de la información, aprender a aprender, autonomía y iniciativa personal), como ya señalábamos en un estudio anterior sobre la utilización de las TIC en trabajos académicos (Martí y Garcia, 2014).

También ha sido muy positiva la valoración del alumnado puesto que las TIC les motivan más a participar en las actividades propuestas en la materia, les facilitan acceder a los contenidos del currículo, interaccionar entre ellos y con las profesoras, y compartir otros actividades de interés cultural, referentes musicales y cinematográficos, vídeos, fotografías, etc.

Por otra parte, las redes sociales posibilitan la escritura colaborativa de manera que los estudiantes interactúan y reflexionan con las herramientas apropiadas, como dice Castelló (2009). Además, estos textos tienen un carácter público que supone un mayor nivel de exigencia en la elaboración y presentación de los trabajos. Por lo tanto, las redes sociales presentan nuevas posibilidades didácticas y su uso académico supone una innovación educativa durante el proceso de aprendizaje del alumnado que se debería tener más en cuenta en la enseñanza superior.

En este estudio hemos revisado ejemplos de trabajos académicos con el uso de redes sociales que el alumnado valora muy positivamente para mejorar el trabajo colaborativo y la motivación en proyectos de lengua y literatura. Consideramos importante, pues, favorecer la difusión de este tipo de trabajos que ponen en evidencia las ventajas del uso de las redes sociales con el fin de contribuir a aumentar la interacción entre el alumnado y el profesorado, el aprendizaje de las diferentes habilidades lingüísticas y la difusión de trabajos académicos más allá de los límites del aula y del entorno educativo.

(cc) EY-NC-ND 2018, Universitat Politècnica de València

Congreso IN-RED (2018) 


\section{Referencias}

ÁreA MoreirA, M. (2008). La innovación pedagógica con TIC y el desarrollo de las competencias informacionales y digitales. Investigación en la Escuela, 64, 5-17. $<$ http://www.investigacionenlaescuela.es/articulos/64/R64_1.pdf $>$ [Consulta: 8 de septiembre de 2017]

CAstelló, M. (2009). Aprender a escribir textos académicos: ¿copistas, escribas, compiladores o escritores?. En J.I. Pozo; Pérez Echevarría, M.P. (coords.) Psicología del aprendizaje universitario: la formación en competencias. Madrid: Morata, 120-133.

García GarcíA, D. (2014). Creación y edición de vídeo. En Trujillo, F. (coord.) Artefactos digitales. Una escuela digital para la educación de hoy. Barcelona: Graó, 114-117.

GARCiA VidAl, P.; MARTí Climent, A. (2010). Les xarxes socials com a recurs educatiu. Quaderns Digitals.net, 65.

Garcia Vidal, P.; Martí Climent, A. (2016). Treballem llengua i literatura amb les TIC. Aplicació didàctica en la formació del futur professorat de secundària. Quaderns digitals.net, 82 .

Gómez, M.; Roses, S.; Farias, P. (2012). El uso académico de las redes sociales en universitarios. Comunicar. Revista Científica de Comunicación y Educación. DOI: 10.3916/C38-2011-03-04, <https:/www.revistacomunicar.com/pdf/preprint/38/14-PRE13426.pdf> [Consulta: 23 de octubre de 2017]

HARO, J.J.. (2009). Las redes sociales aplicadas a la práctica docente. Didáctica, Innovación y Multimedia, 13, 1-8.

HARO, J.J. (2010). Redes Sociales para la educación. Madrid: Anaya.

HARO, J.J. (2010). Las redes sociales en educación. $<$ http://jjdeharo.blogspot.com.es/2008/11/la-redes-sociales-en-educacin.html $>$ [Consulta: 18 de septiembre de 2017]

Lluch, G. (2012). La lectura al Centre. Llegir (i escriure) llibres, pantalles, documents al Pla de Lectura de Centre. Alzira: Editorial Bromera.

LLUCH, G. (2014). Jóvenes y adolescentes hablan de lectura en la red. Ocnos, 11, 7-20. $<$ https://www.revista.uclm.es/index.php/ocnos/article/view/ocnos_2014.11.01> [Consulta: 27 de noviembre de 2017]

Lluch, G.; Nicolás, M. (2015). Escriptura acadèmica. Planificació, documentació, redacció, citació i models. Barcelona: UOC.

MACÍAS, C. (2016). Las redes sociales como herramientas de aprendizaje: algunas consideraciones. Revista de Estudios Latinos (RELat) 16, 225-256.

MAnReSA, M.; Duran, C. I RAMADA, L. (2012). Les TIC en les seqüències didàctiques de llengua i literatura, en Projectes de treball per ensenyar i aprendre llengua i literatura. Articles de Didàctica de la Llengua i la Literatura, 57, 36-48.

MARTí Climent, A. (2018). Booktubers en valencià. [en prensa]

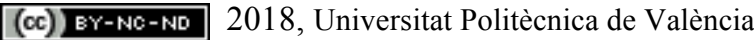
Congreso In-Red (2018) 
Martí Climent, A. ; Garcia Vidal, P. (2014). El treball per projectes amb les TIC a l'àrea de Llengua i Literatura. Una experiència didàctica en la formació del professorat de Secundària. Articles de Didàctica de la Llengua i la literatura, 64.

Meso Ayerdi K.; Pérez Dasilva J. Y.; Mendiguren Galdospin, T. (2011). La implementación de las redes sociales en la enseñanza superior universitaria. Tejuelo, 12 (2011), 137-155.

ORIHUELA, J. L. (2009). Redes sociales y educación. $<$ http://www.ecuaderno.com/2009/03/10/redes-sociales-y-educacion/ $>$ [Consulta: 15 de enero de 2018]

PÉREZ GARCíA, A. (2013). Redes Sociales y Educación. Una reflexión acerca de su uso $\begin{array}{llllll}\text { didáctico } & \mathrm{y} & \text { creativo. } & \text { Creatividad } & y & \text { sociedad, }\end{array}$ $<$ http://www.creatividadysociedad.com/articulos/21/12.\%20Redes $\% 20$ Sociales $\% 20 y \% 20 \mathrm{e}$ ducacion. $\% 20$ Una $\% 20$ reflexion $\% 20$ acerca $\% 20 \mathrm{de} \% 20 \mathrm{su} \% 20 \mathrm{uso} \% 20$ didactico $\% 20 \mathrm{y} \% 20 \mathrm{cre}$ ativo.pdf> [Consulta: 26 de febrero de 2018]

PRENSKY, M. (2001). Digital Natives, Digital Immigrants. On the Horizon. MCB University Press, Vol. 9 No. 5. <https://www.marcprensky.com/writing/Prensky\%20\%20Digital\%20Natives,\%20Digital\%20Immigrants\%20-\%20Part1.pdf $>$ [Consulta: 15 de octubre de 2017]

Rovira Collado, J. (2015). Redes sociales de lectura: del libro de caras a la LIJ 2.0. Investigaciones sobre Lectura, 3, 106-122. 